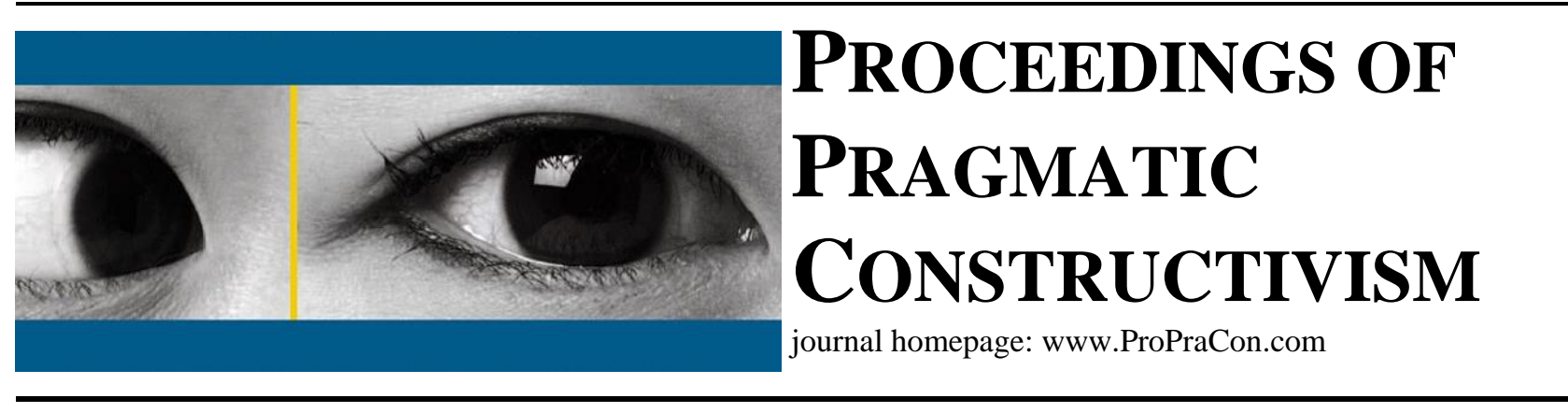

\title{
Are you sure about what you mean by 'uncertainty'? The actor's perspective vs. the institutional perspective
}

\author{
Boris Genadiev Borisov \\ Research Assistant \\ Aarhus University; School of Business and Social Sciences; Department of Economics and Business \\ Fuglesangs Allé 4, 8210 Aarhus V, Denmark; boris.genadiev@gmail.com \\ Rainer Lueg \\ Associate Professor of Management Accounting and Control \\ Aarhus University; School of Business and Social Sciences; Department of Economics and Business \\ Fuglesangs Allé 4, 8210 Aarhus V, Denmark; rlueg@asb.dk
}

\begin{abstract}
This paper explicates theoretical and methodological differences between Archival Environmental Uncertainty (AEU) and Perceived Environmental Uncertainty (PEU). Conceptually, we discuss the controversial development of the concepts in a literature review. We propose a reconciling framework which emphasizes that AEU and PEU differ due to the specificity of the decision unit, the predictability of change, and the use of leading indicators. We conclude that future conceptual work could further refine AEU- and PEU-measures; especially a better distinction between AEU and 'risk' is warranted.

Empirically, we are the first ones to investigate the statistical association between prevailing measures of AEU (Tosi et al., 1973; Dess and Beard, 1984) and PEU (Miller, 1993). Our analysis combines archival data on AEU (annual reports) with survey data on PEU from top executives of the 110 largest listed German companies (55\% response rate) by using time series-, factor- and correlation-analyses. Our findings show-as predicted - that AEU and PEU correlate moderately on a significant level. Yet, adjustment of the AEU-measure for predictable changes does not increase the strength of this correlation. This implies that future empirical work should focus more on the specificity of the decision unit and the use of leading indicators than on adjusting the predictability of change.
\end{abstract}

Keywords: Environmental uncertainty; perceived; archival; methodology; objective; correlation.

Acknowledgement: This paper is part of a larger research project on strategic management control (Burkert \& Lueg, 2013; Lueg, 2008, 2009, 2010a, b; Lueg \& Borisov, 2014; Lueg \& Schäffer, 2010). 


\title{
Are you sure about what you mean by 'uncertainty'? \\ The actor's perspective vs. the institutional perspective
}

\author{
The Actor-Reality Perspective in a Global Economy \\ (Second Conference)
}

Scuola Superiore Sant'Anna, Pisa (I) - Oct $25^{\text {th }}-26^{\text {th }} 2012$

Boris Borisov \& Rainer Lueg | Aarhus University

Uncertainty is the counterpart of risk

Uncertainty vs. risk

- Outcomes unknown

- Distribution unknown

- Many sub-concepts, e.g., Environmental Uncertainty

- Different possible outcomes are known

- Distribution of outcomes are known

- Many sub-concepts 


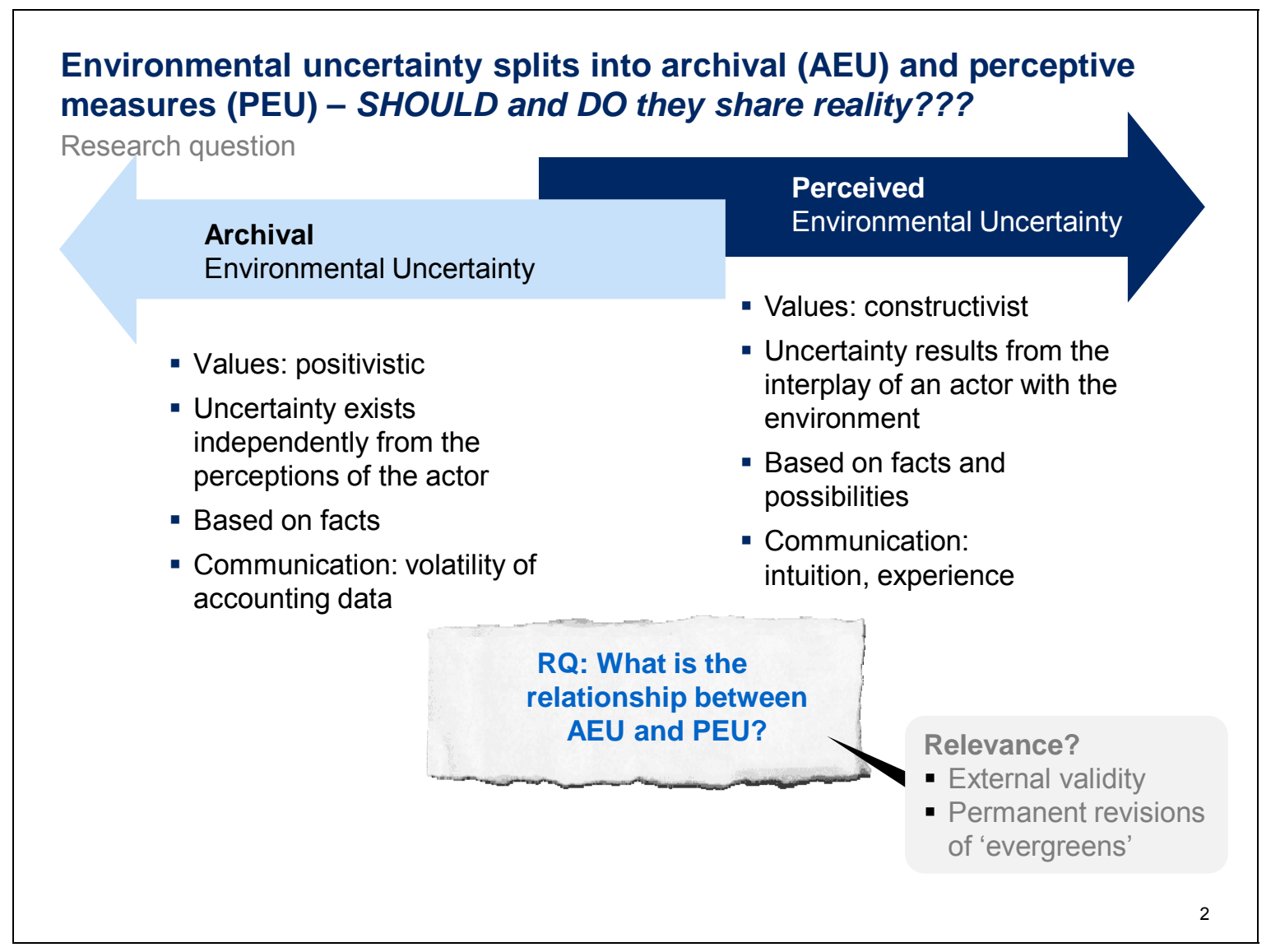

AEU is assessed by accounting measures, PEU by a survey they are both measured at the industry-level

Instruments AEU and PEU

Archival

Perceived

Environmental Uncertainty *

10-year volatility of...

- Sales

- Earnings before interests and taxes (EBIT)

- Employment levels (in fulltime equivalents, FTEs)

- Earnings After Taxes (EAT)

- Equity (book value)

- Total assets
"How predicable is the environment in which your company operates?

- The production/service technology is not subject to very much change and is well established (e.g. steel production).

- Demand and consumer tastes are fairly easy to forecast (e.g. milk industry).

- The rate at which products and services are getting obsolete in the industry is very slow (e.g. commodities like oil).

- Actions of competitors are quite easy to predict (e.g. pure price competition)." 


\section{Based on the conceptualization, the relationship should be significant, but only moderately strong}

Hypotheses 1: The relationship between AEU and PEU

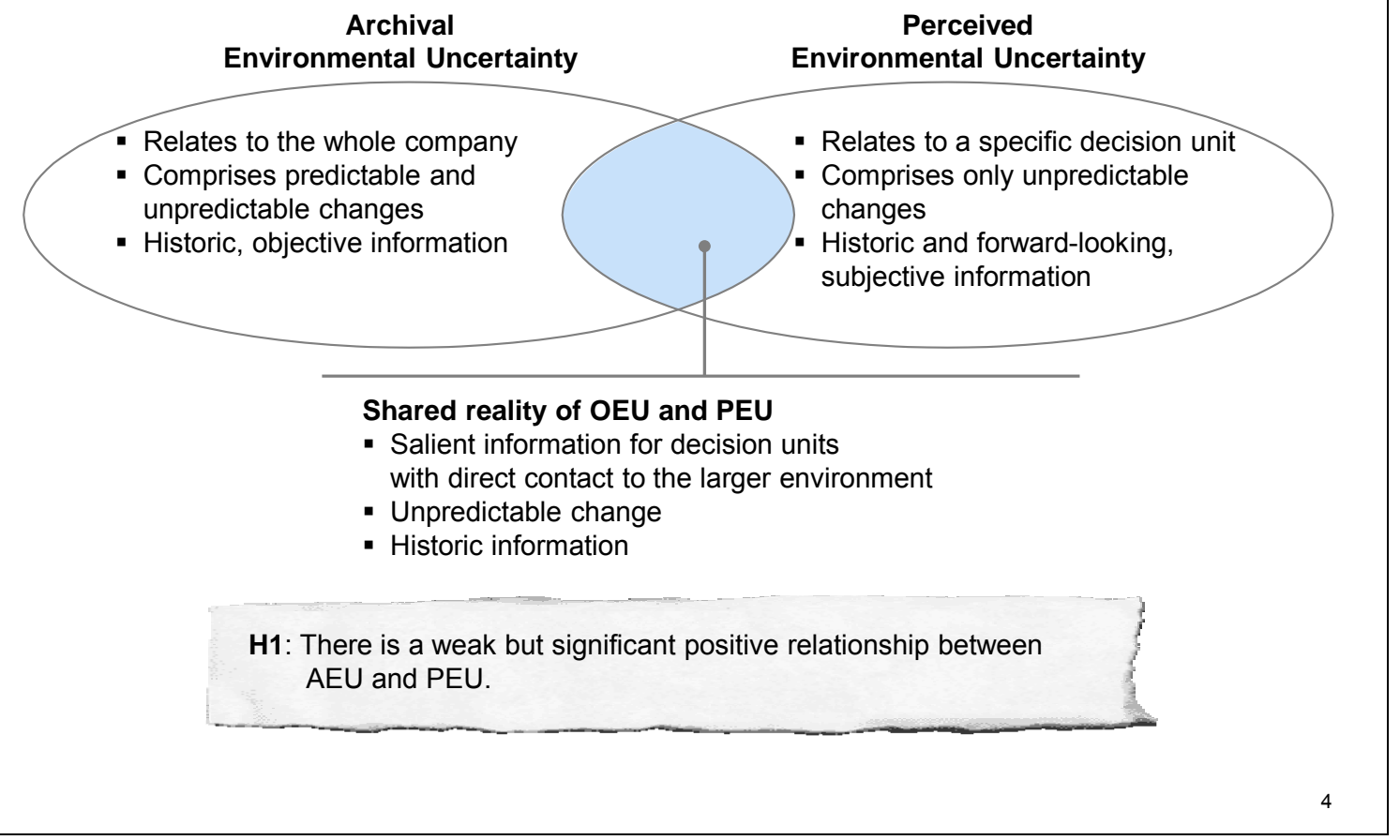

Evidence on the relationship of AEU and PEU is inconclusive

Hypothesis 2: Adjustments in AEU (1/2)

No relationship

- Tosi et al. (1973, p. 31) vs. Lawrence \& Lorsch (1967): "low and inconsistent correlation".

- Snyder \& Glueck (1982, p. 191) vs. Tosi et al. (1973) : "biasing effect of individual differences".
Relationship

- Sharfman \& Dean (1991, p. 689) vs.

Dess \& Beard (1984): "in general, the

correlations were significant and in the proper direction"

- Karimi, Sommers \& Gupta (2004) with Miller (1993) vs. Dess \& Beard (1984) "The results further challenge the notion that CEOs perceptions are inclined to be imprecise, erroneous."

\section{Suggested solution}

"It is not change per se, or even a fast rate of change, that creates uncertainty about the environment; rather, it is unpredictable change that will be associated with this type of uncertainty. Thus, a lack of correlation between measures of environmental volatility and perceived environmental uncertainty is not, in and of itself, reasonable grounds for claiming that the perceptual measures are invalid." 
Is 'unpredictable' vs. 'predictable' change the missing link?

Hypothesis 2: Adjustments in AEU (2/2)

\section{Sales variation}

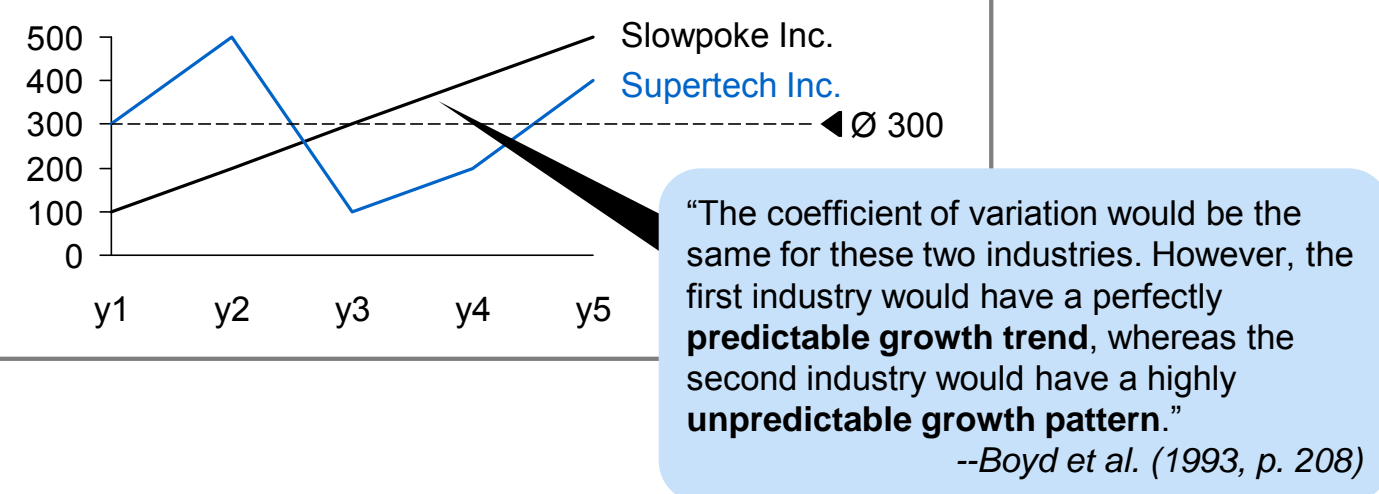

H2: Adjusting* measures of AEU for predictable change will improve the relationship of AEU and PEU.

* Tosi et al (1973): Standard error around the mean (includes predictable trends)

Dess \& Beard (1984): Standard error of 10-year regression line divided by the mean (excludes predictable trends)

Data come from the annual reports and a top management survey of the German HDAX companies

Data source

\begin{tabular}{|c|c|c|c|c|c|c|c|c|c|}
\hline \multicolumn{2}{|c|}{ Employees } & \multicolumn{2}{|c|}{$\begin{array}{c}\text { Sales } \\
\text { (in mio. EUR) } \\
\end{array}$} & \multicolumn{2}{|c|}{$\begin{array}{c}\text { Market } \\
\text { capitalization } \\
\text { (in mio. EUR) } \\
\end{array}$} & \multicolumn{2}{|l|}{$\begin{array}{c}\text { Industry } \\
\text { (1-digit-SIC) }\end{array}$} & \multicolumn{2}{|l|}{$\begin{array}{r}\text { Respondents } \\
\text { (by function) }\end{array}$} \\
\hline$<500$ & 2 & $<500$ & 2 & $<500$ & 8 & Manufacturing & 33 & \multirow{3}{*}{$\begin{array}{l}\text { Accounting, } \\
\text { Control } \\
\text { \& Finance }\end{array}$} & \multirow[t]{3}{*}{34} \\
\hline$<1,000$ & 7 & $<1,000$ & 13 & $<1,000$ & 7 & \multirow{2}{*}{$\begin{array}{l}\text { Finance, insurance, } \\
\text { real estate }\end{array}$} & \multirow[t]{2}{*}{8} & & \\
\hline$<5,000$ & 11 & $<5,000$ & 18 & $<5,000$ & 21 & & & & \\
\hline$<10,000$ & 10 & $<10,000$ & 7 & $<10,000$ & 6 & & & \multirow{2}{*}{$\begin{array}{l}\text { Investor } \\
\text { Relations }\end{array}$} & \multirow[t]{2}{*}{13} \\
\hline$<50,000$ & 14 & $<50,000$ & 15 & $<50,000$ & 14 & Services & 7 & & \\
\hline$<100,000$ & 8 & $<100,000$ & 4 & $<100,000$ & 4 & Trade & 6 & Corporate & 8 \\
\hline$<250,000$ & 4 & $<250,000$ & 1 & & & Transportation \& & 6 & Development & \\
\hline$<500,000$ & 4 & & & & & public utilities & & Executive Board & 5 \\
\hline $\mathrm{n}=$ & 60 & & 60 & & 60 & & 60 & & 60 \\
\hline
\end{tabular}


PEU correlates significantly with almost all AEU measures, and the strength is just moderate

Test H1: Correlations

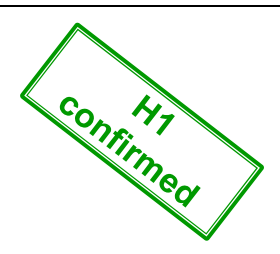

\begin{tabular}{|c|c|c|c|c|c|c|c|c|c|c|c|c|}
\hline No. Variation of: & PEU & $2 a$ & $2 \mathbf{b}$ & $3 a$ & $3 \mathbf{b}$ & $4 a$ & $4 \mathbf{b}$ & $5 a$ & $5 \mathbf{b}$ & $6 a$ & $6 \mathrm{~b}$ & $7 a$ \\
\hline $\begin{array}{l}1 \text { PEU [dynamism] } \\
\text { (Miller, 1993) }\end{array}$ & 1 & & & & & & & & & & & \\
\hline $\begin{array}{l}\text { 2a Sales } \\
\text { (Tosi et al., 1973) }\end{array}$ & $\begin{array}{l}0.374 * * \\
0.002\end{array}$ & 1 & & & & & & & & & & \\
\hline $\begin{array}{l}\text { 2b Sales } \\
\quad \text { (Dess \& Beard, 1984) }\end{array}$ & $\begin{array}{l}0.326 * \\
0.007\end{array}$ & $\begin{array}{l}0.635 * * * \\
0.000\end{array}$ & 1 & & & & & & & & & \\
\hline $\begin{array}{l}\text { 3a EBIT } \\
\text { (Tosi et al., 1973) }\end{array}$ & $0.281 *$ & $\begin{array}{l}0.372 * * \\
0.003\end{array}$ & $\begin{array}{l}0.266 * \\
0.026\end{array}$ & 1 & & & & & & & & \\
\hline $\begin{array}{l}\text { 3b EBIT } \\
\text { (Dess \& Beard, 1984) }\end{array}$ & $\begin{array}{c}0.049 \\
0.370\end{array}$ & $\begin{array}{c}0.230 \\
0.058\end{array}$ & $\begin{array}{l}0.090 \\
0.271\end{array}$ & $\begin{array}{l}0.875 * * * \\
0.000\end{array}$ & 1 & & & & & & & \\
\hline $\begin{array}{l}\text { 4a Employment } \\
\text { (Tosi et al., 1973) }\end{array}$ & $\begin{array}{l}0.353 * * \\
0.004\end{array}$ & $\begin{array}{l}0.785 * * * \\
0.000\end{array}$ & $\begin{array}{l}0.491 * * * \\
0.000\end{array}$ & $\begin{array}{l}0.186 \\
0.089\end{array}$ & $\begin{array}{l}0.120 \\
0.208\end{array}$ & 1 & & & & & & \\
\hline $\begin{array}{l}\text { 4b Employment } \\
\text { (Dess \& Beard, 1984) }\end{array}$ & $\begin{array}{l}0.192 \\
0.078\end{array}$ & $\begin{array}{l}0.236 * \\
0.040\end{array}$ & $\begin{array}{l}0.273 * \\
0.021\end{array}$ & $\begin{array}{l}0.216 \\
0.059\end{array}$ & $\begin{array}{l}0.101 \\
0.247\end{array}$ & $\begin{array}{l}0.445 * * * \\
0.000\end{array}$ & 1 & & & & & \\
\hline $\begin{array}{l}\text { 5a Earnings } \\
\text { (Tosi et al., 1973) }\end{array}$ & $\begin{array}{l}0.277 * \\
0.025\end{array}$ & $\begin{array}{c}0.063 \\
0.330\end{array}$ & $\begin{array}{l}0.303 \\
0.015\end{array}$ & $\begin{array}{l}0.360 * * \\
0.005\end{array}$ & $\begin{array}{l}-0.136 \\
0.187\end{array}$ & $\begin{array}{r}0.050 \\
0.363\end{array}$ & $\begin{array}{l}0.171 \\
0.115\end{array}$ & 1 & & & & \\
\hline $\begin{array}{l}5 \mathbf{b} \text { Earnings } \\
\text { (Dess \& Beard, 1984) }\end{array}$ & $\begin{array}{l}0.257 * \\
0.040\end{array}$ & $\begin{array}{r}-0.032 \\
0.416\end{array}$ & $\begin{array}{l}0.182 \\
0.110\end{array}$ & $\begin{array}{l}0.329 * \\
0.013\end{array}$ & $0.402 * *$ & $\begin{array}{l}-0.010 \\
0.473\end{array}$ & $\begin{array}{l}0.131 \\
0.190\end{array}$ & $\begin{array}{l}0.894 * * * \\
0.000\end{array}$ & 1 & & & \\
\hline $\begin{array}{l}\text { 6a Equity } \\
\text { (Tosi et al., 1973) }\end{array}$ & $\begin{array}{l}0.313 * * \\
0.010\end{array}$ & $\begin{array}{l}0.670 * * * \\
0.000\end{array}$ & $\begin{array}{l}0.220 \\
0.053\end{array}$ & $\begin{array}{l}0.280 * \\
0.021\end{array}$ & $\begin{array}{l}0.052 \\
0.363\end{array}$ & $\begin{array}{l}0.645 * * * \\
0.000\end{array}$ & $0.342 * *$ & $\begin{array}{l}0.066 \\
0.325\end{array}$ & $\begin{array}{l}-0.077 \\
0.303\end{array}$ & 1 & & \\
\hline $\begin{array}{l}\text { 6b Equity } \\
\text { (Dess \& Beard, 1984) }\end{array}$ & $\begin{array}{l}0.360 * * \\
0.004\end{array}$ & $\begin{array}{l}0.423 * * \\
0.001\end{array}$ & $\begin{array}{l}0.179 \\
0.097\end{array}$ & $0.312 *$ & $\begin{array}{l}-0.290 * \\
0.024\end{array}$ & $\begin{array}{l}0.391 * * \\
0.002\end{array}$ & $0.379 * *$ & $\begin{array}{l}0.184 \\
0.103\end{array}$ & $\begin{array}{c}0.033 \\
0.413\end{array}$ & $\begin{array}{l}0.707 * * * \\
0.000\end{array}$ & 1 & \\
\hline $\begin{array}{l}\text { 7a Assets } \\
\text { (Tosi et al., 1973) }\end{array}$ & $\begin{array}{l}0.268 * \\
0.023\end{array}$ & $\begin{array}{l}0.742 * * * \\
0.000\end{array}$ & $\begin{array}{l}0.244 * \\
0.035\end{array}$ & $\begin{array}{l}0.303 * \\
0.013\end{array}$ & $\begin{array}{l}0.178 \\
0.113\end{array}$ & $\begin{array}{l}0.684 * * * \\
0.000\end{array}$ & $\begin{array}{l}0.407 * * \\
0.001\end{array}$ & $\begin{array}{l}-0.024 \\
0.435\end{array}$ & $\begin{array}{r}-0.132 \\
0.189\end{array}$ & $\begin{array}{l}0.744 * * * \\
0.000\end{array}$ & $\begin{array}{l}0.455 * * * \\
0.000\end{array}$ & 1 \\
\hline $\begin{array}{l}\text { 7b Assets } \\
\text { (Dess \& Beard, 1984) }\end{array}$ & $0.303 *$ & $\begin{array}{l}0.638 * * * \\
0.000\end{array}$ & $\begin{array}{l}0.461 * * * \\
0.000\end{array}$ & $\begin{array}{l}0.420 * * \\
0.001\end{array}$ & $\begin{array}{l}-0.015 \\
0.460\end{array}$ & $\begin{array}{l}0.577 * * * \\
0.000\end{array}$ & $\begin{array}{l}0.516 * * * \\
0.000\end{array}$ & $\begin{array}{l}0.155 \\
0.141\end{array}$ & $\begin{array}{l}0.026 \\
0.433\end{array}$ & $\begin{array}{l}0.629 * * * \\
0.000\end{array}$ & $\begin{array}{l}0.784 * * * \\
0.000\end{array}$ & $0.612 * * *$ \\
\hline
\end{tabular}

Both AEU measures explain PEU well - Yet, the trend-adjusted measure of Dess \& Beard (1984) does not outperform Tosi et al. (1973)

Test H2: Regression*; dependent variable is PEU (Miller, 1993)

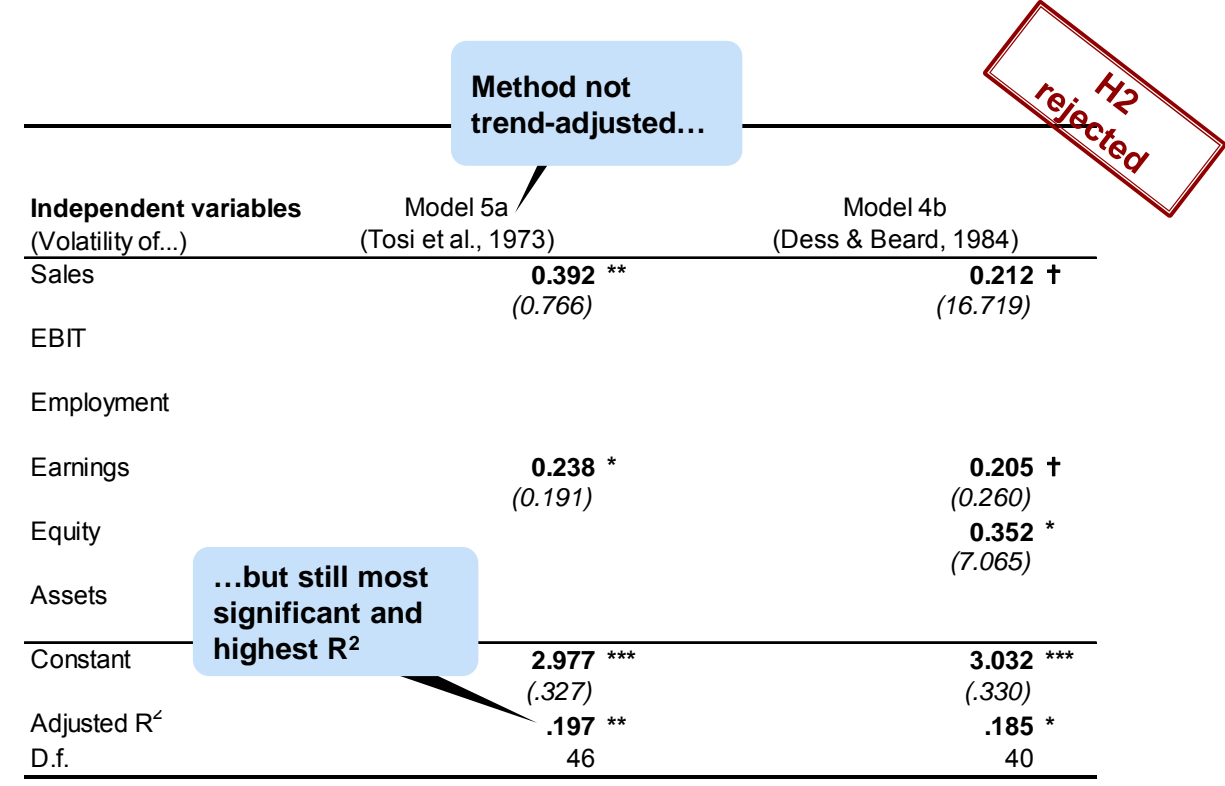




\section{AEU and PEU are not perfect substitutes,} but valid proxies at the level of the industry I top executives

Implications

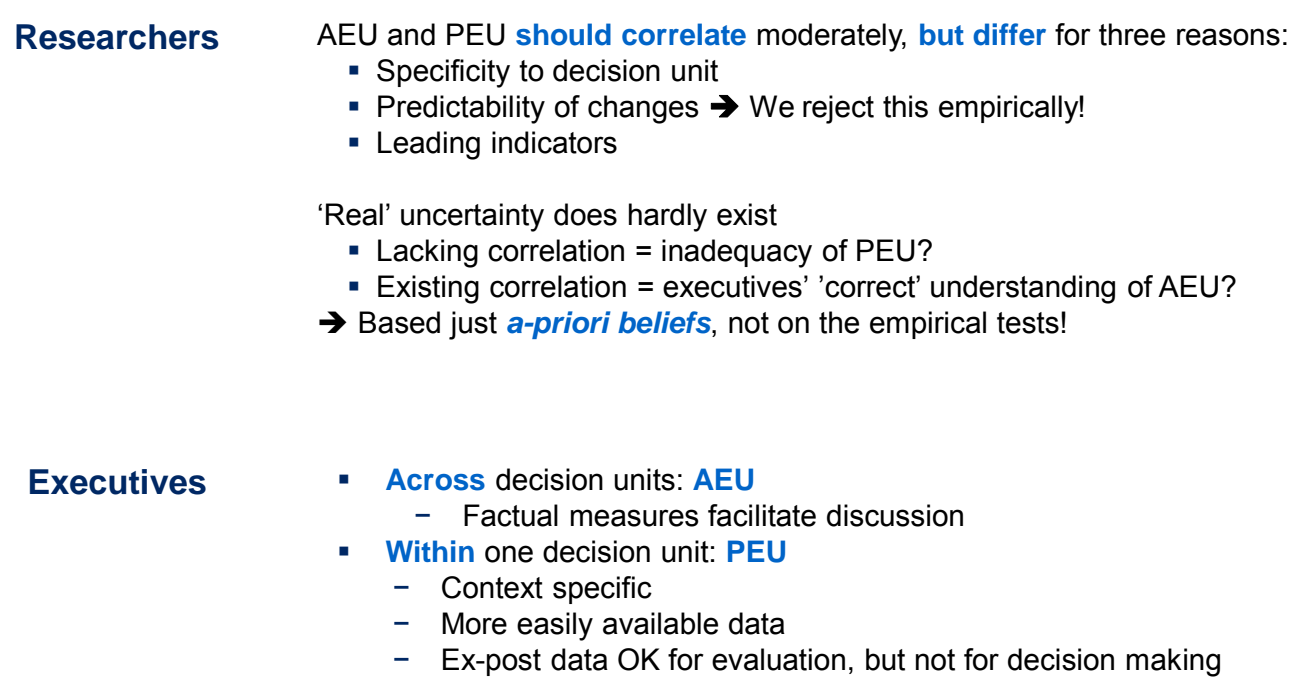

We just investigate how facts and possibilities create shared realities Future research should critically challenge values and communication

Values

- Rethink old measures of AEU/PEU

- Adjust uncertainty to context

- Look at other levels (hierarchies, functions, society)

- Clarify the difference of AEU and risk

\section{Communication}

- Do executives use different types of information on uncertainty (AEU, PEU) for different purposes like scanning, decision making, control and evaluation?

- How do external stakeholders (define and) communicate uncertainty, e.g., shareholders, banks, analysts or rating agencies? 


\section{References}

Bourgeois, L. J. 1985. Strategic goals, perceived uncertainty, and economic performance in volatile environments. Academy of Management Journal, 28(3): 548-573.

Boyd, B. K., Dess, G. G., \& Rasheed, A. M. A. 1993. Divergence between archival and perceptual measures of the envrionment: causes and consequences. Academy of Management Review, 18(2): 204-226.

Burkert, M., \& Lueg, R. 2013. Differences in the sophistication of Value-based Management - The role of top executives. Management Accounting Research, 24(1): 3-22.

Chenhall, R. H. 2003. Management control systems design within its organizational context: findings from contingency-based research and directions for the future. Accounting, Organizations and Society, 28(2-3): 127168.

Dess, G. G., \& Beard, D. W. 1984. Dimensions of organizational task environments. Administrative Science Quarterly, 29(1): 52-73.

Downey, H. K., Hellriegel, D., \& Slocum Jr, J. W. 1975. Environmental uncertainty: the construct and its application. Administrative Science Quarterly, 20(4): 613-629.

Downey, H. K., Hellriegel, D., \& Slocum, J. W. 1977. Individual characteristics as sources of perceived uncertainty variability. Human Relations, 30(2): 161-174.

Duncan, R. B. 1972. Characteristics of organizational environments and perceived environmental uncertainty. Administrative Science Quarterly, 17(3): 313-327.

Lueg, R. 2008. Value-based Management: Empirical Evidence on its Determinants and Performance Effects. Vallendar: WHU Otto Beisheim School of Management.

Lueg, R. 2009. Führt der Einsatz externer Berater zur Überimplementierung innovativer Steuerungsinstrumente? Zeitschrift der Unternehmensberatung, 4(6): 249-253.

Lueg, R. 2010a. Shareholder Value und Value Based Management - Wie steuern die HDAX-Konzerne? Zeitschrift für Controlling, 22(6): 337-344.

Lueg, R. 2010b. Value-based Management - Antecedents and performance effects. In K. Pantz (Ed.), Summa Cum Laude 2008: Wirtschaftswissenschaften: 284-285. Darmstadt: Roter Fleck Verlag

Lueg, R., \& Borisov, B. G. 2014. Archival or perceived measures of environmental uncertainty? Conceptualization and new empirical evidence. European Management Journal: forthcoming.

Lueg, R., \& Schäffer, U. 2010. Assessing empirical research on Value-based Management: guidelines for improved hypothesis testing. Journal für Betriebswirtschaft, 60(1): 1-47.

Miller, D. 1993. Industry and country effects on managers' perceptions of environmental uncertainties. Journal of International Business Studies, 24(4): 693-714.

Milliken, F. J. 1987. Three types of perceived uncertainty about the environment: state, effect, and response uncertainty. Academy of Management Review, 12(1): 133-143.

Tosi, H., Aldag, R., \& Storey, R. 1973. On the measurement of the environment: an assessment of the Lawrence and Lorsch environmental uncertainty subscale. Administrative Science Quarterly, 18(1): 27-36. 